\title{
The Design and Implementation of a Batteryless Wireless Embedded System for loT Applications
}

\author{
Mehmet Erkan Yüksel \\ Department of Computer Engineering, Mehmet Akif Ersoy University, Burdur, Turkey
}

Cite this article as: Yüksel ME. Design and Implementation of A Batteryless Wireless Embedded System for loT Applications. Electrica, 2019; 19(1): 1-11.

\begin{abstract}
With the increasing popularity of embedded systems that consist of identifying, sensing, processing, and communication capabilities, the Internet of Things (IOT) has enabled many new application scenarios in diverse scientific fields and provided a significant opportunity to build efficient industrial systems and applications. Most embedded systems consume power provided by fixed batteries with limited capacity. However, the main disadvantage of batteries is that they must be periodically replaced with new ones or recharged when they are depleted. This kind of maintenance process increases the cost and restricts the use in inaccessible places. When considering the limitations of battery power, alternative energy sources are required for interconnected devices to operate efficiently and effectively. Harvesting or scavenging energy from the environment is an important strategy to design self-powered systems employed in the loT domain. Traditional energy harvesting applications use large energy storage devices to supply the power to the system whenever needed. Batteryless energy harvesting techniques have advantages to operate long periods without maintenance. In this study, we designed and implemented a supercapacitor-based, solar-powered wireless embedded system that can operate autonomously without the need of maintenance and battery replacement. Our goal is to explore and analyze the applicability of batteryless wireless data communication and the usefulness of self-powered smart devices employing an energy harvesting technique in the loT environment. In our experiments, we observed that our prototype boards operate well with low-power consumption without any performance degradation.
\end{abstract}

Keywords: IoT, wireless communication, embedded systems, solar energy, energy harvesting, supercapacitor

\section{Corresponding Author:}

Mehmet Erkan Yüksel

E-mail:

erkanyuksel@mehmetakif.edu.tr

Received: 03.05.2018

Accepted: 30.07 .2018

(C) Copyright 2019 by Electrica

Available online at

http://electrica.istanbul.edu.tr

DOI: 10.26650/electrica.2018.28092

\section{Introduction}

Internet of Things (IoT) is a rapidly developing technology that has a wide range of application domains, technological innovations, and social impacts. It is a dynamic global network infrastructure consisting of interconnected devices with identification, sensing, data processing, information exchange, and communication capabilities. It provides an intelligent ecosystem for the information society by enabling advanced services, standards, and platforms.

With recent advances in wireless communications, digital electronics, integrated circuits (IC), sensors, and micro-electro-mechanical systems (MEMS) technology, the design and development of low-cost, low-power, multifunctional and autonomous wireless smart devices have become important.The ever-increasing capabilities of these devices enable the realization of various IoT applications based on the collaborative effort of a large number of smart devices [1-3].

The general architecture and basic components of a wireless smart device are shown in Figure 1. It can be an embedded system or a single-board computer to perform multiple tasks. It typically consists of a sensing unit to sense changes in its surroundings; a communication unit to interact with other devices or the Internet to take an active role in the decision-making process; a computing unit to process information; and a power source. Moreover, several components such as an actuator, an external memory, a location finding system, and a power generator can also be integrated into the device depending on the loT application.

Sophisticated hardware and software features place additional challenges on the operation of wireless smart devices. Wireless smart devices are battery dependent, and power con- 


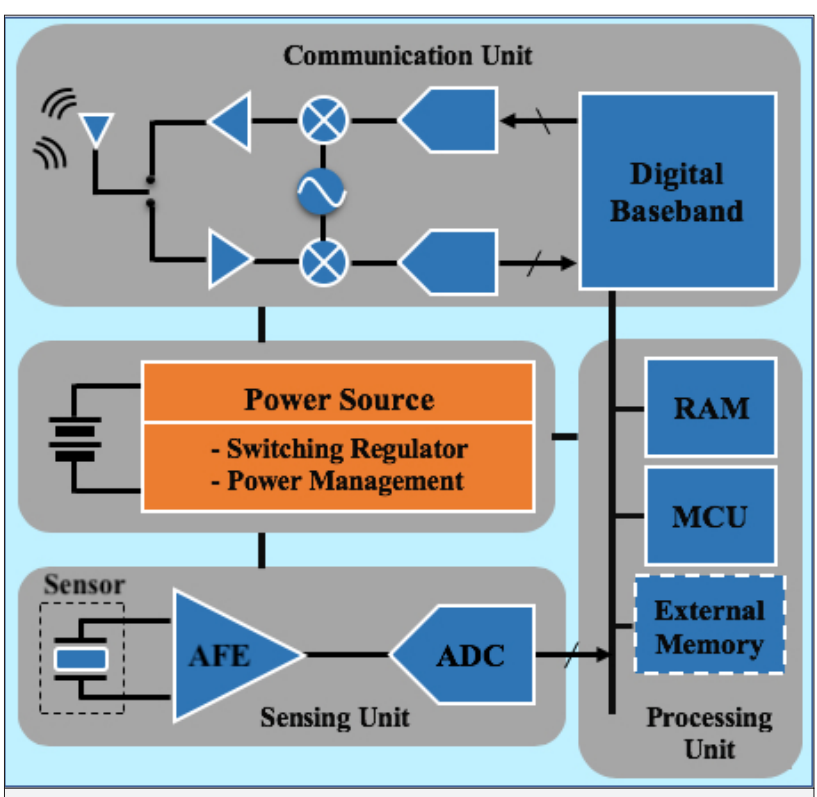

Figure 1. Hardware components of an embedded system

sumption is one of the main factors which significantly influence their design. For most loT applications (i.e., smart home, remote monitoring and control systems, smart city), these devices must operate autonomously and unattended, and must be adaptive to the environment. It may not be feasible to replace or recharge their batteries. With the limited battery power, the operational lifetime of these devices is also limited. Therefore, power is a scarce and vital resource in the IoT ecosystem. It is possible to extend the operational lifetime of wireless smart devices through energy harvesting techniques. Solar cells provide practical and cost-effective solutions for energy-efficient operations. However, the amount of energy that can be extracted is still limited. Consequently, since wireless smart devices are required to perform their tasks efficiently for long periods of time, harvesting or scavenging energy from the environment is an important strategy to design and develop self-powered systems $[4,5]$.

\section{Related Work}

Autonomous embedded systems with low-power consumption have recently received great attention in the literature due to their applications in many emerging technologies such as home automation, smart cities, smart manufacturing, healthcare, wearables, automotive, precision agriculture, supply chain management, and industrial internet. These applications employ a network infrastructure with limited power sources and need to incorporate various energy harvesting systems for their continuous operations.

Various self-sustaining power supplies for wireless embedded systems have been introduced in the past. In [6], the authors presented a solar-harvesting, energy-wise upgradeable platform for environmental sensor networks. The device contains a solar panel as its power source and rechargeable $\mathrm{NiMH}$ batter- ies as its energy storage element. Its design features are simple hardware structure and cheap energy storage units. The authors study the charging and discharging characteristics of the power supplies. In [7], the authors presented the design, implementation, and performance evaluation of a plug-and-play solar energy harvesting module that powers the Berkeley/Crossbow sensor motes. The module has a solar energy harvesting circuit and a rechargeable battery. It autonomously manages all decisions related to energy harvesting, energy storage, and power routing. It enables harvesting-aware operation by providing instantaneous solar and battery-state information. In [8], the authors designed a platform to build outdoor sensor networks for environmental monitoring and agriculture applications. The platform is equipped with the Nordic nRF905 RF transceiver, two rechargeable NiMH batteries as the primary power source, a solar panel, an on-board battery charging circuit and regulator, a real-time clock, a flash memory, and a temperature sensor. It is capable of monitoring battery voltage, battery current, charging voltage, and charging current. The power input stage of the platform provides the ability to compute both the energy coming in and the energy being consumed. This enables the implementation of distributed energy-aware applications. In [9], the authors proposed a technique consisting of a power distribution switch (to route power sources to subsystems) and a power source-power consumption matching algorithm (to maximize the total utility of the available power from the ambient power sources). The system is composed of sensors that detect the ambient energy intensity, the controller that runs an algorithm to maximize the utilization of power sources, and the switch array that establishes the connections between power sources and subsystems. The higher utility of ambient power is obtained by combining the maximum power point tracking (MPPT) and the power defragmentation. Moreover, the microcontroller control based on the light sensors is also required. In [10], the authors designed a wireless sensor node to show the feasibility of energy harvesting for sub-watt, small-scale systems integrated with durable energy storage in the form of supercapacitors. They proposed a feed-forward, pulse frequency modulated regulator that charges supercapacitors at near-optimal operating points for solar cells. The device stores energy obtained from solar cells in supercapacitors. It employs an MPPT circuit that enables solar cells to efficiently charge supercapacitors (to maximize the amount of available energy). However, the MPPT circuit requires a microcontroller to run the MPPT algorithm. In [11], the authors developed a system that manages energy transfer for perpetual operation (to maximize the lifetime of the sensor device). Their platform uses a solar panel, a charging circuit, and a two-stage storage unit consisting of supercapacitors (a primary buffer charged by a solar panel to power the device) and a Li+ rechargeable battery (a secondary buffer as a reliable emergency backup. It is charged either by solar panel or by the primary buffer).

\section{System Design and Implementation}

In our study, we developed a supercapacitor-operated, solar-powered, autonomous wireless embedded system for loT 
applications. The hardware architecture of our system is depicted in Figure 2.

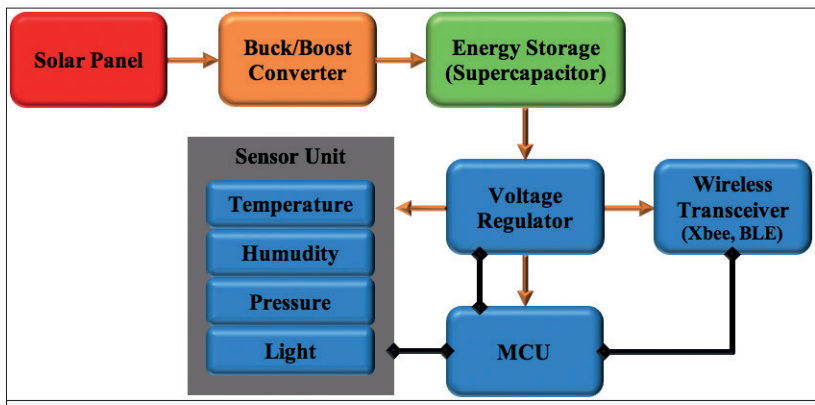

Figure 2. Embedded system architecture

The system is designed for three main tasks: using a solar panel as a power source, charging the supercapacitor through a switching regulator, and performing energy management for all typical sensor and wireless communication functions.

In order to obtain the maximum power from the solar panel (6V 150mA), a buck/boost converter is used. A supercapacitor is chosen instead of a rechargeable battery to store harvested energy. Because, a supercapacitor has infinite charging cycles, and its charging/discharging efficiency is higher than that of a battery. When the available energy in the supercapacitor is insufficient to operate the converter, the initial energy harvesting process is passive. Once enough energy is stored for the reliable startup of the converter, the system transitions to an operational state where it actively manages the energy harvesting and controls the power availability to the embedded device. If the energy stored in the supercapacitor is enough to supply power to the embedded device, then the system enables the device to operate with regulated voltage, while continuing to harvest energy from the solar panel Finally, a microcontroller (Atmega328P) is employed to perform several tasks, such as processing sensor data, energy management, and communication.

Our embedded device provides information about its surrounding environment to a host system through its XBee or BLE module (i.e., BLE supported devices such as smartphones can obtain information from the device). In addition, the application running on the device uses an energy management software to optimize the energy efficiency (or usage). Consequently, all components of our device interact with each other, and therefore, each component influences the energy transfer between them, as well as the power consumption and behavior of the complete system.

Figure 3 shows the implementation of our batteryless wireless embedded system and prototype devices. All of the devices operate autonomously when the solar panel and supercapacitor are integrated with the devices.

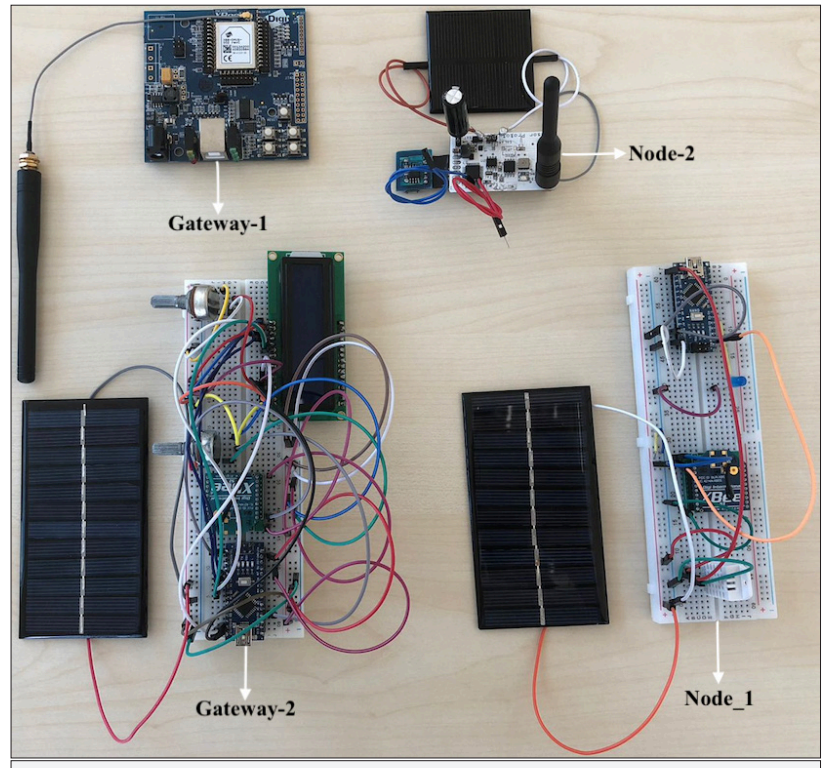

Figure 3. Our prototype boards and development tools

\section{Power source}

Solar is an important energy source for autonomous embedded systems, usually due to the following factors:

- Solar energy is clean, sustainable, renewable, and inexhaustible. It is readily available anywhere.

- Solar cells can produce electricity from available light even under low-irradiance conditions (i.e., indoor environments) [12]. They provide high power density compared to other power sources such as acoustic noise, thermal, radio frequency, vibrations, airflow.

- $\quad$ Photovoltaic (PV) systems have advantages such as easy implementation, high reliability, silent operation, long life, low maintenance cost, and employing simple equipments with no moving or rotating parts $[12,13]$.

- Many embedded systems use solar cells in recent years, because solar energy provides sufficient power for most of today's smart electronic devices that consume up to several hundred $\mathrm{mW}$ of power $[2,8,12-17]$.

Solar cells generate electricity from sunlight. They are connected together in either series or parallel combinations or both to form a solar module/panel which provides higher output voltages and currents. If solar cells are connected in series the voltage increases. If they are connected in parallel, then the current increases (Figure 4). An energy harvesting circuit fixes the output power of the solar cell and transfers this power to the energy storage unit. It performs avoiding the backflow from the energy storage unit to the solar cell, protecting the energy storage unit from overload, fixing the operating point for the solar cell [13]. The amount of power generated by the solar cell depends on solar irradiation, the physical and chemical char- 


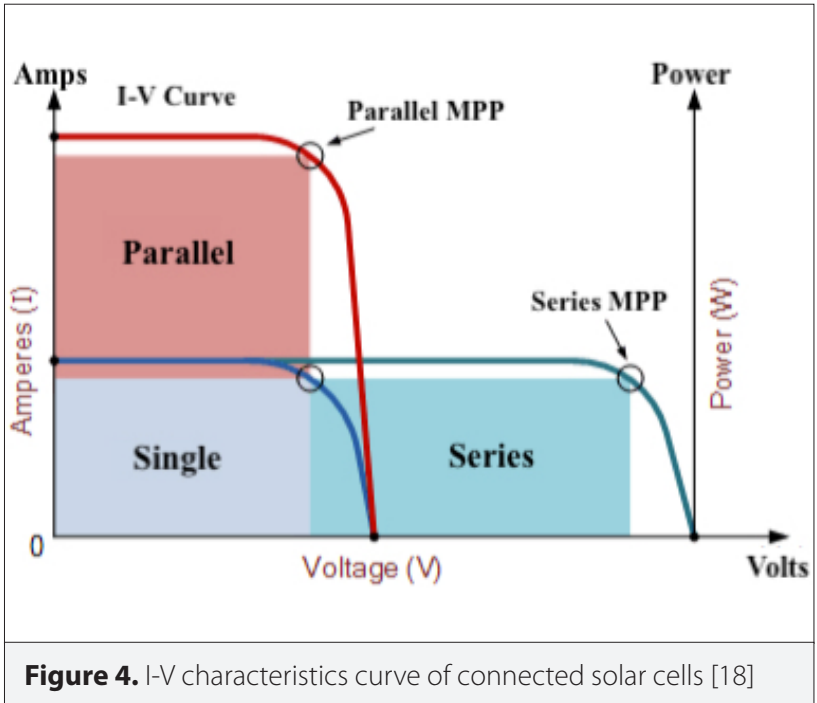

acteristics of the solar cell, the operating point of the solar cell, and weather conditions [13].

To understand the behavior of a solar cell, we model the solar cell by using an equivalent circuit shown in Figure 5 .

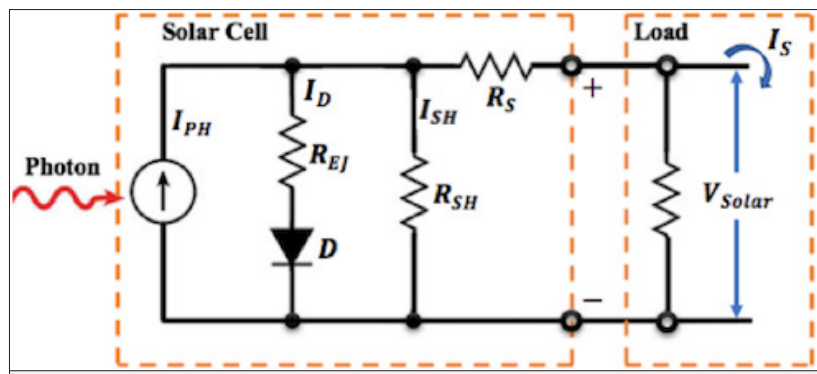

Figure 5. Equivalent circuit of a solar cell

$I_{P H^{\prime}}$ : photogenerated current $I_{S}$ : solar current

$I_{D}$ : diode current $\quad R_{S H}$ : shunt resistance

$I_{s H}:$ shunt current $R_{S}$ : series resistance

$R_{E}$ : equivalent junction resistance

Solar cells produce a current, $I_{P H^{\prime}}$ depending on the incident photons. For instance, considering the $n$ parallel-connected solar cells, $I_{P H}$ is:

$I_{P H}=n \cdot I_{S C}$

$I_{S C}:$ short-circuit current

The exact value of the $I_{S C}$ depends on the operational condition of the solar cell (i.e., cell temperature, solar irradiation, physical and chemical properties of the pn-junction). In addition, the performance of a solar cell can be characterized by its open-cir- cuit voltage $\left(V_{o C}\right)$.

$$
V_{O C} \approx \frac{\eta k T}{q} \ln \left(\frac{I_{P H}}{I_{0}}+1\right), *\left\{I_{P H} \approx I_{S C}\right\}
$$

* for a quality solar cell (high $R_{S H^{\prime}}$ low $R_{S}$ and $I_{O}$ )

As shown in Figure 6, the I-V characteristic curve defines the current and voltage characteristics of a specific solar cell by providing a detailed information about the solar energy conversion ability and efficiency. The I-V characteristics of a solar cell (particularly $P_{\max }$ ) is crucial to analyze the performance and solar efficiency of the embedded system.

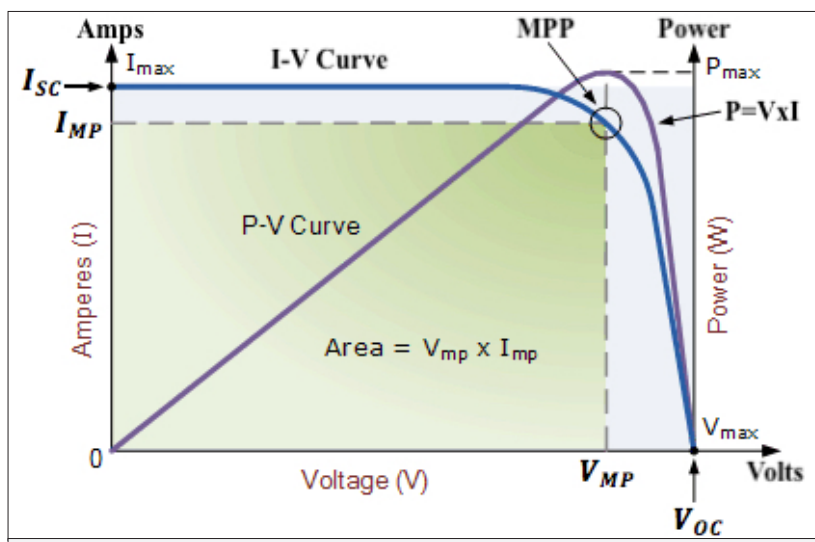

Figure 6. -V characteristics curve of a solar cell [18]

- The current produced by the solar cell:

$$
I_{S}=I_{P H}-I_{D}-I_{S H}
$$

- The output voltage of the solar cell:

$$
V_{S o l a r}=V_{O T}+I_{S} \cdot R_{S}
$$

$V_{O T}$ : voltage across the output terminals

- The diode current:

$$
I_{D}=I_{0}\left(\exp \left[\frac{V_{\text {Solar }}}{\eta \cdot V_{T}}\right]-1\right)
$$

$V_{T}=k T / q$, thermal voltage $\left(\approx 0.0259 \mathrm{~V}\right.$ at $\left.25^{\circ}\right)$

$I_{0}$ : reverse saturation current

$n$ : diode ideality factor

$q$ : electron charge constant

\section{$k$ : Boltzmann constant}

$T$ : absolute temperature 
- The characteristic equation of the solar cell:

$$
I_{S}=I_{P H}-I_{0}\left(\exp \left[\frac{V_{\text {Solar }}}{\eta \cdot V_{T}}\right]-1\right)-\frac{V_{\text {Solar }}}{R_{S H}}
$$

*The generated power through the solar cell:

$$
P_{\text {Solar }}=V_{\text {Solar }} \cdot I_{S}
$$

The I-V characteristics of solar cells are non-linear and non-monotonic. Depending on each solar irradiance level, the power reaches a global maximum at a certain voltage (or current), which is the optimal operating point (Maximum Power Point-MPP) to obtain the maximum amount of power from the solar cell (Figure 7).
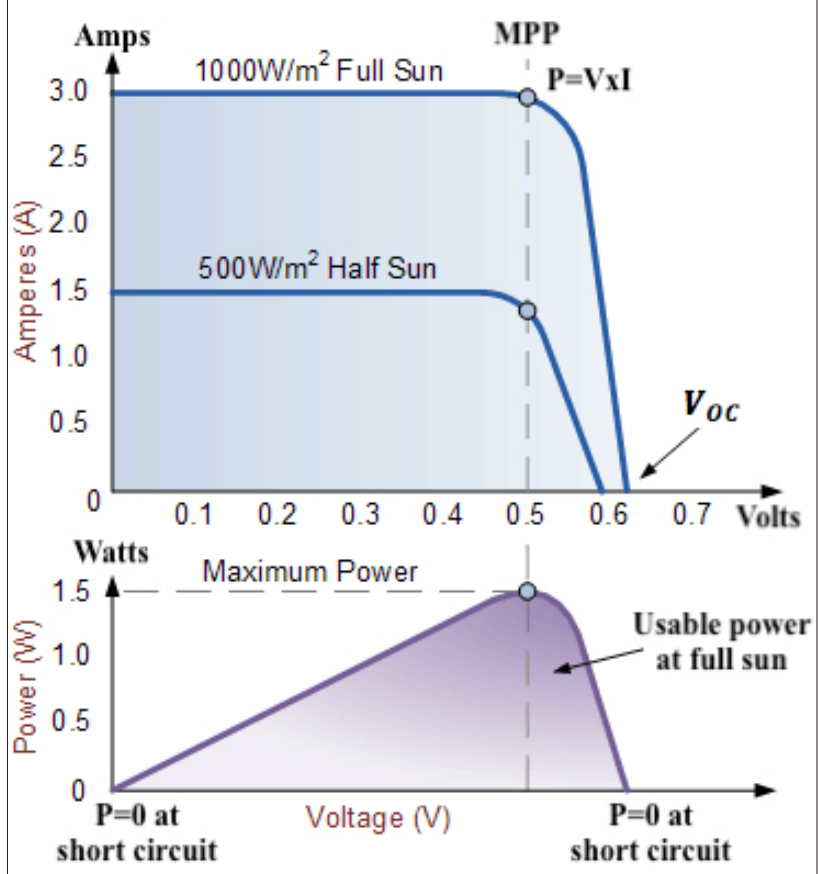

Figure 7. I-V characteristics curve in response to sunlight [18]

The MPP is calculated by the equations below:

$$
\begin{aligned}
& \frac{d P_{\text {Solar }}}{d V_{\text {Solar }}}=\frac{d P_{\text {Solar }}}{d I_{\text {Solar }}}=0 \\
& \frac{I_{P H}+I_{0}}{I_{0}}=\left(\frac{q \cdot V_{M P P}}{\eta k T}+1\right) \cdot \exp \left(\frac{q \cdot V_{M P P}}{\eta k T}\right)
\end{aligned}
$$

As can be seen from Eq. 9, the MPP can be referred to as a function of $I_{P H}$ and $I_{O^{\prime}}$ which depends on the solar irradiance and temperature. $V_{M P P}$ represents the output voltage of the solar cell when the solar cell is operating at the MPP. Eq. 9 can provide a basis for MPP tracking (MPPT). However, the implementation of MPPT in energy harvesting systems is a challenging task be- cause of the need to measure the voltage and current levels of solar cells, temperature, and solar irradiance. Therefore, several MPPT techniques have been proposed in the literature to obtain more accurate results [19-22]. The power generated by a solar panel at a $W_{S l}$ solar irradiance is given below:

$$
P_{\text {Solar }}=\min \left(P_{R} \cdot \frac{W_{S I}}{W_{R}}, P_{\text {Solar }}^{m A x}\right)
$$

$P_{R}:$ rated power output of the solar cell

$W_{R}:$ rated irradiance level

\section{Energy storage}

It is crucial to choose a well-suited storage device for embedded systems. Many of today's embedded systems use batteries as energy storage. However, batteries are often stated as a major limiting factor that influences the operational lifetime of embedded systems. Batteries have a high energy density; however, they have limited power density (charge-discharge rates). In addition, continuous high rate cycling, inability to hold a full charge for a long time, and harsh environmental conditions (i.e., temperature) adversely affect both battery performance and lifetime. These negative effects lead to periodic maintenance and battery replacement for embedded systems. Unfortunately, such recurring maintenance and replacement can be very expensive if they must be done for many devices, which are likely to be time-consuming, expensive, and difficult to access after deployment. Consequently, the battery is a crucial factor which prevents the embedded systems from operating maintenance-free for more than several years at different data rates [23].

One solution which provides the long-term operation of embedded systems without the need of a battery is to use a supercapacitor. The viable technique is to remove the battery altogether, replace it with the supercapacitor and store energy in it. Supercapacitors are a good choice for embedded systems due to their operational features, particularly the high power density. They have high charge-discharge efficiency, unlimited charging cycles, wide operating temperature range, and long lifetime (Table 1). They do not release any thermal heat during

Table 1. Comparison of supercapacitors and batteries

\begin{tabular}{lcc}
\hline Property & Supercapacitors & Batteries \\
\hline Energy density & Low & High \\
\hline Power density & High & Low \\
\hline Voltage behavior & Varying & Constant \\
\hline Cycle life & High & Low \\
\hline Temperature peformance & Good & Poor \\
\hline DC life & High & Medium \\
\hline Form factor & Small & Large \\
\hline Weight & Light & Heavy \\
\hline
\end{tabular}


the discharging process. They are also known as a green technology because of the high efficiency and the materials used in production. These outstanding features make supercapacitors a useful energy storage device for embedded systems employed in loT [24-28].

Supercapacitors have advantages in terms of energy storage. A general idea about the performance of these devices in comparison with normal capacitors and batteries can be obtained by examining their situations on the Ragone plot shown in Figure 8. The Ragone plot describes the relationship between the energy density and power density of various energy storage/energy propulsion technologies. Thus, it

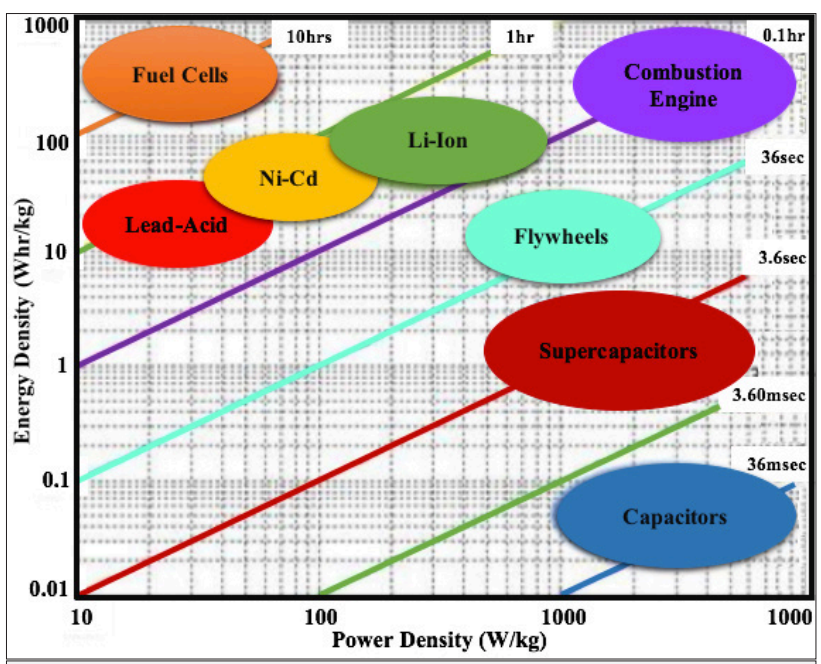

Figure 8. Performane comparison of energy storage devices is feasible to analyze the device's ability to store energy for long periods (high energy density) against its ability to obtain a large amount of energy in a short time when required (high power density). As shown in Figure 8, supercapacitors are located between capacitors and batteries, depending on their characteristic features. They have higher power density than batteries and higher energy density than normal capacitors. They are not exposed to chemical reactions; therefore, they have a much higher number of charge cycles.

\section{Energy harvesting}

As shown in Figure 9, our energy harvesting system uses a DC/DC switching regulator that has a wide range of input and output voltages, and high conversion efficiency over the embedded device's operating power range. The regulator is placed between the solar cell and supercapacitor to increase the harvesting efficiency. It prevents the supercapacitor from degrading the operation performance of the solar cell. Such a structure enables energy harvesting to continue even when $V_{O C}<V_{\text {SCOP }}$. The regulator also serves as a diode to block reverse current flow from supercapacitor to the solar cell [29].

Wireless embedded systems operate at a constant input voltage. Therefore, the energy storage device rarely has the same voltage. A boost (step-up) converter is employed to increase the input voltage. In the case of a higher storage voltage, a buck (step-down) converter is more efficient. Our choice is to use a buck/boost DC/DC converter that enables up-down voltage conversion to operate the embedded system independent of the supercapacitor voltage efficiently. The reg-

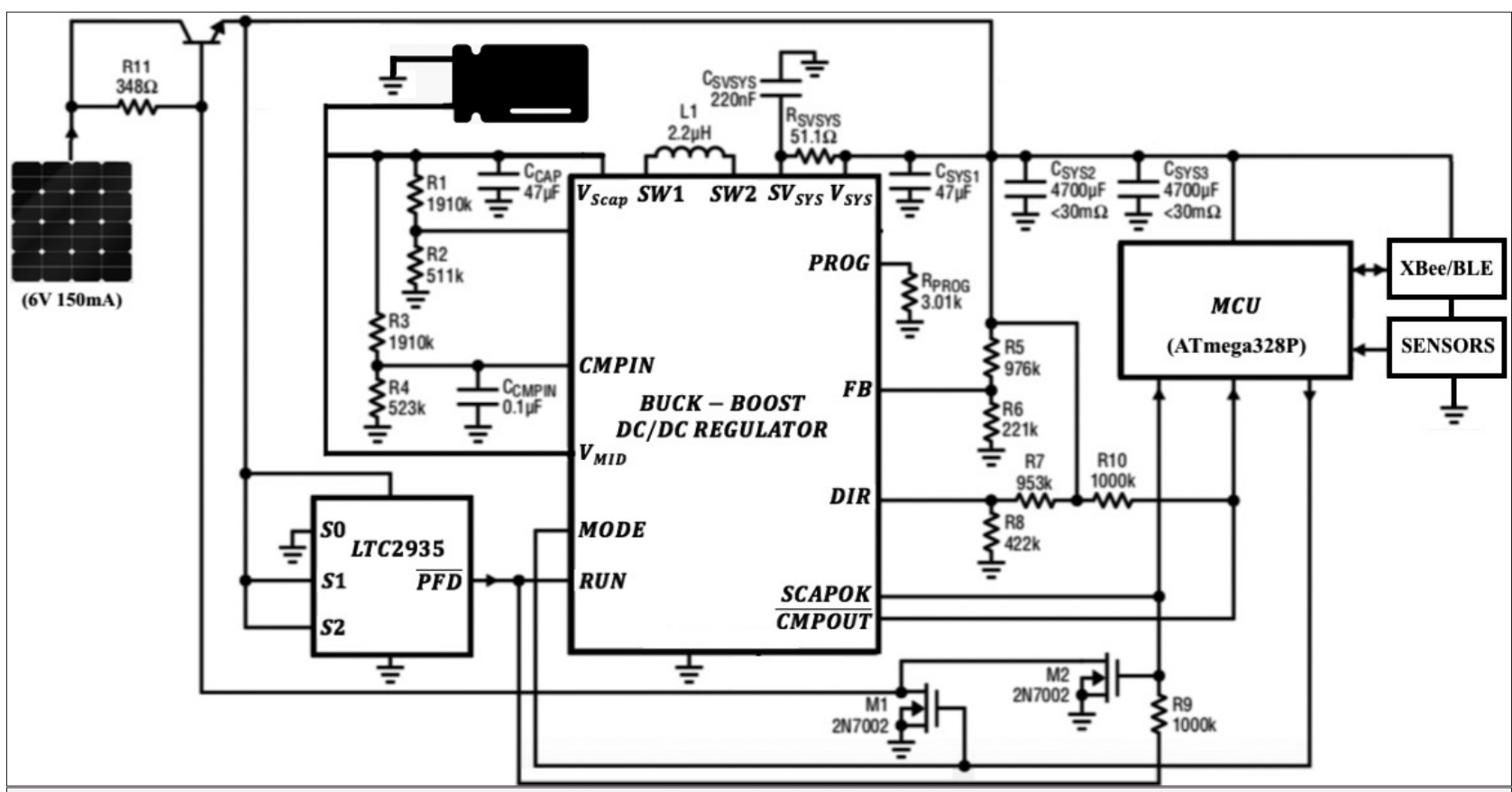

Figure 9. Implementation of the energy harvesting system 
ulator is set to operate at the lowest possible voltage level to improve the energy efficiency of the embedded system. In addition, the operating voltage is adjusted by an energy management software using dynamic power management technique to reduce system power consumption without degrading performance. Finally, a secondary switching regulator is employed to operate MCU, wireless transceiver, and sensors, which require different voltage than the embedded system's voltage [27-29].

\section{Energy harvesting method}

Power management techniques designed for the energy-efficient operation of embedded systems differ significantly when the power source changes from a battery to an energy harvesting module. One of the reasons which leads to differences is that the ambient energy source is highly variable. Contrary to the stored energy characterized by the amount of the battery's residual energy, ambient energy requires a more sophisticated characterization. Another reason is that ambient energy is not a scarce resource and has the potential to be used forever for the sustainable performance of the embedded systems.

Since the ambient energy source varies a great deal, the main design goal is to increase the overall system performance. Another goal is to determine whether ambient energy is enough for the embedded system to perform its tasks at the desired performance level. If so, the system operates efficiently and effectively. If not, energy harvesting requirements must be specified for the system. Consequently, the aim of developing an energy harvesting technique is to enable an analysis of various energy harvesting technologies with respect to system performance [28-30].
To obtain better results on the performance and lifetime of wireless embedded systems operating from an available energy source, it is necessary to specify the important features/parameters of the energy availability. This situation requires modeling the energy provided by a harvesting system that converts ambient energy into electrical energy and stores it in a supercapacitor. We can make use of a model proposed in [27] to characterize the production and consumption of ambient energy for our prototype board, which employs an energy harvesting system shown in Figure 10 .

- The power output of the energy source:

$E_{\text {Source }}\left(P, e_{1}, e_{2}\right): \begin{aligned} & \int_{T} E_{S}(t) d t \geq P T-e_{1} \\ & \int_{T} E_{S}(t) d t \leq P T+e_{2}\end{aligned}$

-The power consumption of the energy consumer:

$$
E_{\text {Consumer }}\left(P^{\prime}, e\right): \int_{T} E_{C}(t) d t \leq P^{\prime} T+e
$$

In the above definitions, $E_{s}\left(P, e_{1}, e_{2}\right)$ denotes the energy source (solar panel), which supplies power to our embedded system. $E_{s}(t)$ models the power output of the energy source at time $t . E_{c}$ $\left(P^{\prime}, e\right)$ denotes the energy consumer (embedded system), which obtains power from the energy source. $E_{c}(t)$ models the power consumption of the embedded system at time $t . P$ and $P^{\prime}$ are the available powers at which the system can operate. The variables $\left(e_{1} e_{1}, e_{2}\right)$ denote the energy storage capacity of the embedded system.

Figure 10 shows the general architecture of our energy harvesting system, which describes the energy flow in our prototype board. The solar panel produces a current, $l_{\text {solar }} \geq 0$, depending on ambient conditions. The switching regulator (with capacitor charger and balancer features) converts the input voltage to an

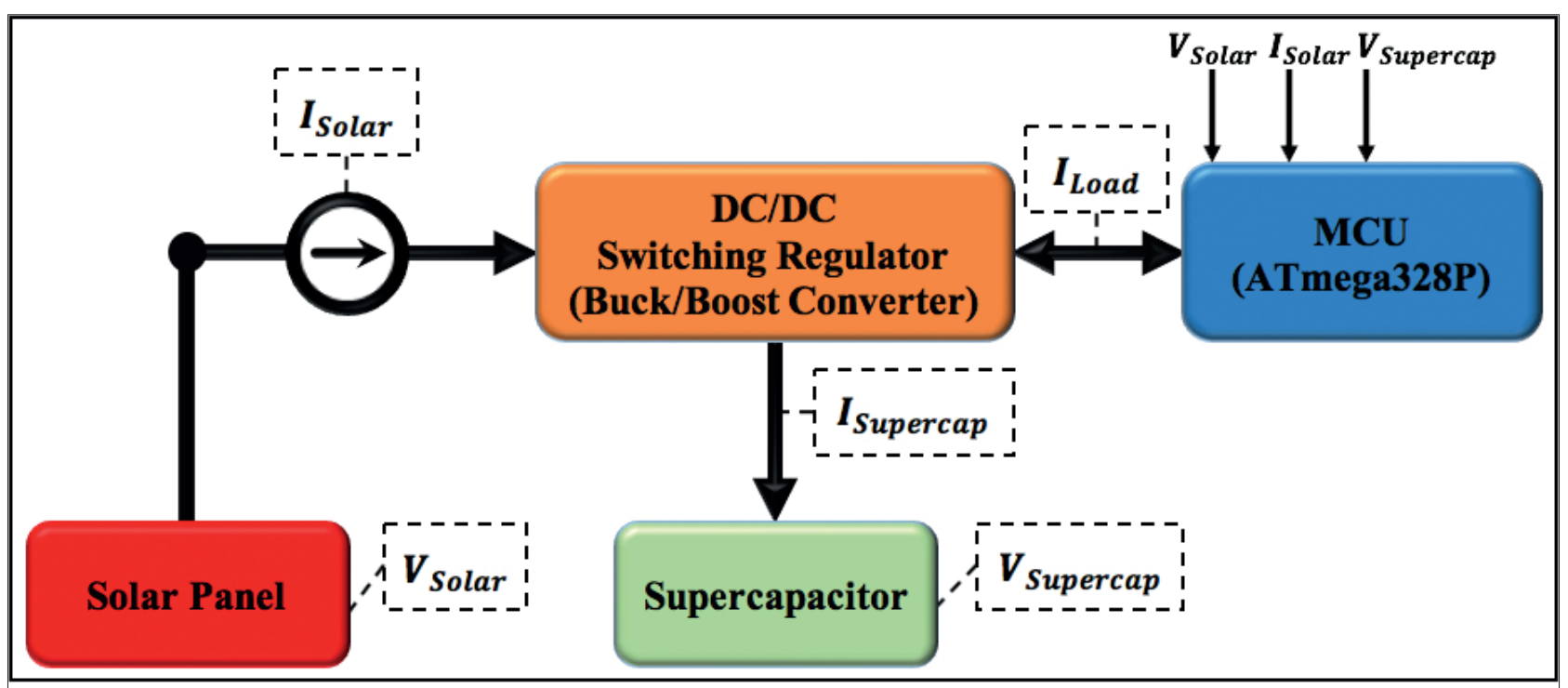

Figure 10. Block diagram of our energy harvesting system 
output voltage with high efficiency (Figure 11). The regulator current, $I_{\text {Reg }}>0$, is consumed by the regulator to supply the embedded system with the current $I_{\text {Load }}>0$ at the output voltage $V_{\text {Load }}$. The supercapacitor serves as energy storage. The current $I_{\text {scap }}$ flows into the supercapacitor with a capacity of $C$. If $I_{\text {scap }}>0$, the supercapacitor is charged; otherwise, it acts as a power source and discharges to supply the system. The supercapacitor voltage, $V_{\text {scap }}$ cannot exceed $V_{\text {max }}$ because of overcharging protection. If $V_{\text {scap }}$ underruns the cut-off voltage $V_{\text {cutoff }}$ then the regulator fails. Finally, the power-conversion efficiency of the regulator, $n$, is a function of $I_{\text {Load }}$ and $V_{\text {scap }}$.

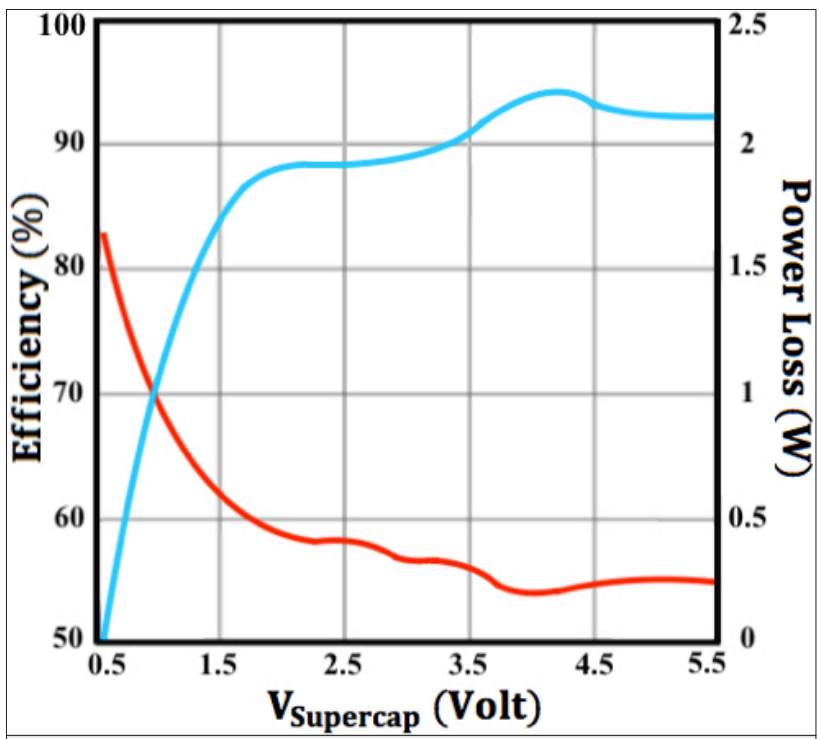

Figure 11. Charge efficiency of the switching regulator

Based on the information mentioned above, we can model the energy flow for our embedded system as follows:

$$
\begin{aligned}
& I_{\text {Solar }}=I_{\text {Reg }}+I_{\text {Scap }} \\
& I_{\text {Reg }}=\frac{V_{\text {Load }} \cdot I_{\text {Load }}}{V_{\text {Scap }} \cdot \eta \cdot\left(V_{\text {Scap }}, I_{\text {Load }}\right)}, I_{\text {Scap }}=C \cdot V_{\text {Scap }}
\end{aligned}
$$

The embedded system influences the overall system power consumption by changing its duty cycle. We can model it as a power sink of periodic pulses. In most cases, we only model the average power consumption by assuming the wake time is negligible:

$$
P_{a v g}^{d}=V_{s u p} \cdot\left(d \cdot I_{a c t}+(1-d) \cdot I_{s l p}\right)
$$

where

$P_{a v g}^{d}$ : average power consumption

$V_{\text {sup }}:$ supply voltage

d: duty cycle,

$I_{\text {act }}:$ active mode current

$I_{s t p}:$ sleep mode current

\section{Results and Discussion}

For autonomous wireless embedded systems with limited battery power employed in loT, continuous power availability and low maintenance cost are crucial. Energy harvesting technologies and batteryless systems have the advantage of being able to operate for long periods of time in loT applications where the battery replacement is difficult/unsuitable. In addition, wireless technologies such as Zigbee, XBee, LoRa, and BLE have become prominent low-power wireless solutions for both embedded systems and IoT. In order to perform tasks for long periods of time, two approaches can be considered. One is to increase the battery capacity. The other is to reduce system power consumption. The first method increases cost and battery size. It also requires maintenance and battery replacement. To make the system more efficient as well as to reduce the cost and maintenance, it is important to produce low-power types of equipment. There is also one more approach in which batteries are not used. In this method, we have proposed an energy harvesting system consisting of a solar panel, a supercapacitor, a switching regulator, and an energy management software.

In our study, we analyzed how well will wireless technologies, most particularly BLE and XBee, function when employed in a batteryless embedded system that runs on harvested energy and low-power consumption. The generated power is in proportion to the size of the energy harvesting module. In addition, most harvesting systems include energy storage devices to store extra energy. This is used later to meet demand depending on the lack of power when the ambient energy source is temporarily unavailable (i.e., during nights). Energy storage devices can be batteries, supercapacitors, or their combinations. Consequently, our goal is to develop a solar-powered batteryless wireless embedded system considering the low-power consumption and small form factor. We designed a prototype board utilizing a solar panel to harvest energy, a buck/boost DC/DC converter to distribute power, and a supercapacitor to eliminate the battery requirement. Figure 12 shows the performance evaluation of our embedded device with the BLE module at different operation stages.

Since the energy harvester uses a DC voltage, an AC voltage

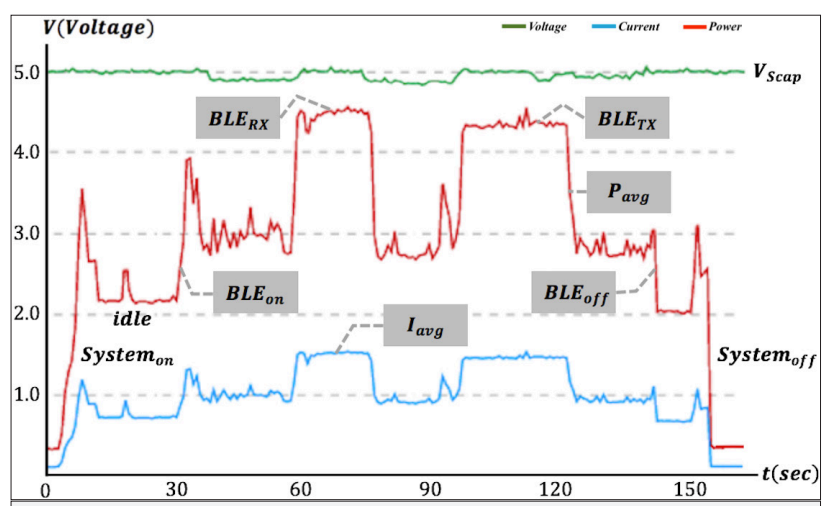

Figure 12. Performance evaluation of the prototype board 
source needs a rectifier before the harvester use AC source. In addition, voltage ratings of the power source highly depend on environmental factors. When the voltage level exceeds the allowed harvester features, a voltage limiter is used before the power source is integrated with energy harvester. Our energy harvesting module employs a switching regulator, and its software executes a power monitoring function to obtain a sufficient amount of energy from the power source.

Wireless modules used in embedded systems consume a large amount of energy while sending and receiving data. Therefore, an optimization technique for the communication system has a crucial impact on the self-sustainability of embedded systems. Except for sending and receiving modes, we can remarkably reduce the power consumption by using the sleep mode that exists in most RF chips. To choose the right RF chip is important in order to obtain low power in the sleep mode for embedded systems.

The microcontroller is another component that consumes more power. It controls all peripheral devices on the board. The most influential part for the power consumption of the microcontroller is the operation clock. Speeding up of the clock increases power consumption. The clock control is a critical factor in reducing power consumption. It is important to select a microcontroller that consumes less power as much as possible in sleep mode and keeps it in sleep mode as long as possible. Another critical situation is the wake-up time from sleep mode. Until the crystal oscillator is stabilized, the microcontroller consumes power during the standby mode. Therefore, the short wake up time is essential to reduce power consumption.

\section{Conclusion}

IoT has become a paramount technology for the information society and led to a paradigm shift in many research areas. It provides us an intelligent ecosystem with infinite opportunities where smart interconnected devices communicate and interact with each other and the Internet. Since loT and smart devices have attracted a great deal of attention in recent years, the design of energy-efficient embedded systems has become more important. Embedded systems must perform well in the IoT environment without the possibility of periodic maintenance. The replacement of these devices can be time-consuming very complex and expensive, even when the device itself is of low cost. In addition to achieving their primary goals, they must provide extra functionalities such as energy-efficient operation, self-diagnostics, self-powered, self-sustained, and network compatibility. Moreover, they need to be extremely secure, robust, and reliable. In order to fulfill all mentioned requirements, they need to possess a certain level of intelligence along with a small form factor.

Ambient energy harvesting provides a viable option to supplement the power supply of energy-constrained embedded systems employed in several loT applications where the required operating lifetime is very long, maintenance and battery re- placements are next to impossible. In this study, we analyzed how an energy harvesting aware power management can supply power to an embedded system, further improve the energy usage and system lifetime. We presented an architecture for the perpetual operation of a self-powered embedded system using the ambient energy source. We made use of a model that can be used to characterize the energy source and determine the sustainable performance of the embedded system. We designed and implemented a batteryless wireless prototype board that employs an energy harvesting module consisting of a solar panel, a supercapacitor, and a DC/DC switching regulator. The regulator facilitates energy transfer from the solar panel into the supercapacitor and charges the supercapacitor at near-optimal operating points for the solar panel. The harvesting module sufficiently supplies power to the board when there is not enough sunlight in the environment. We observed that our board efficiently harvests energy while performing sensing, processing, and communication tasks under different weather conditions. Our device is a low-cost solution and easy to use. It has low hardware complexity and low energy demand, which is easily fulfilled by the supercapacitor. Consequently, supercapacitor-operated solar powered embedded systems can be smaller and lighter, and they do not need to be oversized to accommodate high power cycling. They can be adaptable to loT applications and robustly sustain the prolonged operation. They require less monitoring and less sophisticated management systems and ensure maintenance-free operation.

Peer-review: Externally peer-reviewed.

Conflict of Interest: The author have no conflicts of interest to declare.

Financial Disclosure: The author declared that the study has received no financial support.

\section{References}

1. P. Horowitz, W. Hill, "The art of electronics", Cambridge University Press, Cambridge, United Kingdom, 2015.

2. T. Wu, F. Wu, J. M. Redoute, M. R. Yuce, "An autonomous wireless body area network implementation towards loT connected healthcare applications", IEEE Access, vol. 5, pp. 11413-11422, June, 2017. [CrossRef]

3. F. Wu, C. Rudiger, M. R. Yuce, "Real-time performance of a self-powered environmental loT sensor network system", Sensors, vol. 17, no. 2, pp. 282-296, Feb, 2017. [CrossRef]

4. T. Wu, M. S. Arefin, J. M. Redoute, M. R. Yuce, "A solar energy harvester with an improved MPPT circuit for wearable loT applications", in The International Conference on Body Area Networks, Turin, Italy, 2016, pp. 166-170.

5. D. Gunduz, K. Stamatiou, N. Michelusi, M. Zorzi, "Designing intelligent energy harvesting communication systems", IEEE Communications Magazine, vol. 52, no. 1, pp. 210-216, Jan, 2014. [CrossRef]

6. V. Kyriatzis, N. S. Samaras, P. Stavroulakis, H. Takruri-Rizk, S. Tzortzios, "Enviromote: a new solar-harvesting platform prototype for wireless sensor networks", In The 18th IEEE International Symposium on Personal, Indoor and Mobile Radio Communications, Athens, Greece, 2007, pp. 1-5

7. V. Raghunathan, A. Kansal, J. Hsu, J. Friedman, M. B. Srivastava, 
"Design considerations for solar energy harvesting wireless embedded systems", in The 4th IEEE International Symposium on Information Processing in Sensor Networks, CA, USA, 2005, pp. 457-462. [CrossRef]

8. P. Sikka, P. Corke, L. Overs, P. Valencia, T. Wark, "Fleck-A platform for real-world outdoor sensor networks", in The 3rd International Conference on Intelligent Sensors, Sensor Networks and Information, Melbourne, Australia, 2007, pp. 709-714. [CrossRef]

9. C. Park, P. Chou, "Power utility maximization for multi-supply systems by a load-matching switch", in The International Symposium on Low Power Electronics and Design, CA, USA, 2004, pp. 168-173.

10. F. Simjee, P. H. Chou, "Everlast: long-life, supercapacitor-operated wireless sensor node", in The International Symposium on Low Power Electronics and Design, Tegernsee, Germany, 2006, pp. 197-202. [CrossRef]

11. X. Jiang, J. Polastre, D. Culler, "Perpetual environmentally powered sensor networks," in The 4th International Symposium on Information Processing in Sensor Networks, ID, USA, 2005, pp. 463-468.

12. M. Habibzadeh, M. Hassanalieragh, A. Ishikawa, T. Soyata, G. Sharma, "Hybrid solar-wind energy harvesting for embedded applications: supercapacitor-based system architectures and design tradeoffs", IEEE Circuits and Systems Magazine, vol. 17, no. 4, pp. 29-63, Nov, 2017. [CrossRef]

13. M. Hassanalieragh, T. Soyata, A. Nadeau, G. Sharma, "UR-SolarCap: an open source intelligent auto-wakeup solar energy harvesting system for supercapacitor based energy buffering", IEEE Access, vol. 4, pp. 542-557, Jan, 2016. [CrossRef]

14. C.-Y. Chen, P. H. Chou, "DuraCap: a supercapacitor-based, power-bootstrapping, maximum power point tracking energy-harvesting system", in ACM/IEEE International Symposium on Low-Power Electronics Design, TX, USA, 2010, pp. 313-318. [CrossRef]

15. C. Renner, Volker Turau, "CapLibrate: self-calibration of an energy harvesting power supply with supercapacitors", in The 23th International Conference on Architecture of Computing Systems, Hannover, Germany, 2010, pp. 349-358.

16. D. Brunelli, C. Moser, L. Thiele, L. Benini, “Design of a solar-harvesting circuit for batteryless embedded systems", IEEE Transactions on Circuits and Systems I: Regular Papers, vol. 56, no. 11, pp. 25192528, Feb, 2009. [CrossRef]

17. C. Park, P. H. Chou, "AmbiMax: autonomous energy harvesting platform for multi-supply wireless sensor nodes" in The 3rd Annual IEEE Communications Society Conference on Sensor and Ad Hoc Communications and Networks, VA, USA, 2006, pp. 168-177. [CrossRef]

18. Alternative Energy Tutorials (14.07.2018). Available: http://www. alternative-energy-tutorials.com/solar-power/ solar-power.html.

19. F. I. Simjee, P. H. Chou, "Efficient charging of supercapacitors for extended lifetime of wireless sensor nodes", IEEE Transactions on Power Electronics, vol. 23, no. 3, pp. 1526-1536, May 2008. [CrossRef]

20. P. Bhatnagar, R. K. Nema, "Maximum power point tracking control techniques: state-of-the-art in photovoltaic applications", Renewable and Sustainable Energy Reviews, vol. 23, pp. 224-241, July 2013. [CrossRef]

21. H. Islam, S. Mekhilef, N. B. M. Shah, T. K. Soon, M. Seyedmahmousian, B. Horan, A. Stojcevski, "Performance evaluation of maximum power point tracking approaches and photovoltaic systems", Energies, vol. 11, no. 2, pp. 365-389, Feb, 2018. [CrossRef]

22. T. Esram, P. L. Chapman, "Comparison of photovoltaic array maximum power point tracking techniques", IEEE Transactions on Energy Conversion, vol. 22, no. 2, pp. 439-449, June 2007. [CrossRef]

23. S. Kim, K.-S. No, P. H. Chou, "Design and performance analysis of supercapacitor charging circuits for wireless sensor nodes", IEEE Journal on Emerging and Selected Topics in Circuits and Systems, vol. 1, no. 3, pp. 391-402, Sep, 2011. [CrossRef]

24. D. Dondi, A. Bertacchini, D. Brunelli, L. Larcher, L. Benini, “Modeling and optimization of a solar energy harvester system for self-powered wireless sensor networks", IEEE Transactions on Industrial Electronics, vol. 55, no. 7, pp. 2759-2766, July, 2008.[CrossRef]

25. C. Moser, L. Thiele, D. Brunelli, L. Benini, "Adaptive power management for environmentally powered systems", IEEE Transactions on Computers, vol. 59, no. 4, pp. 478-491, Apr, 2010. [CrossRef]

26. C. Bergonzini, D. Brunelli, L. Benini, "Comparison of energy intake prediction algorithms for systems powered by photovoltaic harvesters", Microelectronics Journal, vol. 40, no. 11, pp. 766-777, Nov, 2010. [CrossRef]

27. A. Kansal, D. Potter, M. B. Srivastava, "Performance aware tasking for environmentally powered sensor networks", in Joint International Conference on Measurement and Modeling of Computer Systems, NY, USA, 2004, pp. 223-234. [CrossRef]

28. J. Hsu, S. Zahedi, A. Kansal, M. B. Srivastava, V. Raghunathan, "Adaptive duty cycling for energy harvesting systems", in The International Symposium on Low Power Electronics and Design, Tegernsee, Germany, 2006, pp.180-185. [CrossRef]

29. A. Kansal, M. B. Srivastava, "Energy harvesting aware power management", Wireless Sensor Networks: A Systems Perspective, ch. 9, pp. 1-10, Artech House, MA, USA, 2005.

30. A. Kansal, J. Hsu, S. Zahedi, M. B. Srivastava, "Power management in energy harvesting sensor networks", ACM Transactions on Embedded Computing Systems, vol. 6, no. 4, pp. 1-35, Sep, 2007. [CrossRef] 


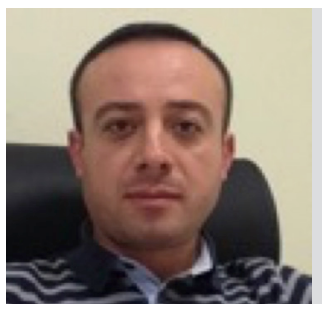

Mehmet Erkan Yüksel received his B.S. degree in Computer Engineering from Firat University in 2007, his MSc and $\mathrm{PhD}$ degrees in Computer Engineering from Istanbul University in 2010 and 2014, respectively. In 2015, he joined the Department of Computer Engineering at Burdur Mehmet Akif Ersoy University, where he is now an Assistant Professor. His research interest includes embedded systems, wireless communications, wireless sensor networks, software defined networks, RFID, agent-based modelling and simulation, artificial intelligence, machine learning, and data mining. 